
\title{
25 Research Soure \\ Thermally Control of Light Propagation in Plasmonics Waveguide and Filter
}

Mehdi Afshari Bavil ( $\nabla$ afsharibawili@gmail.com )

Razi University https://orcid.org/0000-0002-9600-4203

Nader Daneshfar

Razi University

Chuanbo Li

Minzu University of China

Shuai Feng

Minzu University of China

\section{Research Article}

Keywords: Plasmonics, waveguide, filter, vanadium dioxide

Posted Date: May 4th, 2021

DOI: https://doi.org/10.21203/rs.3.rs-436678/v1

License: (c) (1) This work is licensed under a Creative Commons Attribution 4.0 International License.

Read Full License 


\title{
Thermally control of light propagation in plasmonics waveguide and filter
}

\author{
Mehdi Afshari-Bavil ${ }^{1, ~}$, Nader Daneshfar ${ }^{1}$, Chuanbo Li ${ }^{2, \#, ~ S h u a i ~ F e n g ', ~}$ \\ ${ }^{1}$ Department of Physics, Faculty of Science, Razi University, Kermanshah, Iran \\ ${ }^{2}$ School of Science, Minzu University of China, Beijing 100081, China \\ Corresponding author:*afsharibawili@gmail.com,\# cbli@muc.edu.cn
}

\begin{abstract}
An active ultra-compact plasmonic waveguide composed of a subwavelength slit and perforated in vanadium dioxide $\left(\mathrm{VO}_{2}\right)$ followed by a metallic layer is proposed and numerically analyzed. Refractive index variation of $\mathrm{VO}_{2}$ by external stimuli provides a feasible way for tuning the optical properties of the waveguide. Varying the refractive index of $\mathrm{VO}_{2}$ corresponds with changing the phase of $\mathrm{VO}_{2}$ to the metallic state ("on" state). Consequently, the entire structure becomes a typical metal-insulator-metal (MIM) waveguide that routes the incident light through the slit. In addition, during the "off" state, the incident light thereby propagates in the slit and $\mathrm{VO}_{2}$ medium and mitigates rapidly. By adding a MIM waveguide attached to the Fabry-Perot (FP) cavity, spectrally wide stopband and passband filtering features in telecommunication frequency regime are demonstrated. Tailoring the resonance wavelength can be performed through the geometrical parameters. Such active plasmonic waveguides with high transmission, coupling, and compact size can be utilized in future fully integrated all plasmonic chip technology.
\end{abstract}

Keywords: Plasmonics, waveguide, filter, vanadium dioxide.

\section{Introduction}

Plasmonics is a potential device technology to control light in sub-diffraction regime with sufficiently high physical features and minimized geometry $[1,2]$. This natural light squeezing character of surface plasmon beyond the diffraction limit has attracted great interest in developing optical chip minimization with high responsivity and low energy consumption [3-8]. Various developed plasmonic devices such as waveguides [9-12], photodetectors [5, 13-14], modulators [15-18], filters [19-22], and lensing [23-24] have been numerically and/or experimentally demonstrated. For light propagation, different types of wave-guiding structures have been introduced. One of the popular wave-guiding configurations is metal-insulator-metal (MIM) structure that is compatible with the complementary metal-oxide-semiconductor platform [1].

However, modulating light propagation in different conditions by external stimuli to achieve a plasmonic chip is challenging. Tremendous progress has been accomplished in achieving active plasmonic devices, particularly waveguides and filters [25-27]. Tunable surface plasmon polariton (SPP) devices by using liquid crystals, electro optical materials, shape memory alloys, photoacoustic materials, liquid metals, piezo-electric materials, and phase change materials (PCM) have been widely demonstrated. PCM materials play a vital role because of their distinct status under various irritants, such as heat, strain, voltage, optical pumping, and current.

Volatile PCMs such as vanadium dioxide $\left(\mathrm{VO}_{2}\right)[28,29]$ which switches between two recognizable insulators and a metallic phase on a picosecond scale, have a superior characteristic in the telecommunication regime compared with nonvolatile PCMs such as GeSbTe, which switches between crystalline and amorphous states [30]. $\mathrm{VO}_{2}$ has sharp variation in its refractive index when 
undergoing transition from insulator to the metallic phase [31]. The transition occurs when $\mathrm{VO}_{2}$ is agitated by a stimulus, which increases the temperature beyond the phase-transition temperature of $68{ }^{\circ} \mathrm{C}$ [32]. Considering that the $\mathrm{VO}_{2}$ layer is a film without further implementation, the agitation straightforwardly can be accomplished by direct heating to increase the temperature. The transition is reversible on a picosecond time scale. Quantifying this transition is introduced by the mechanism based on Peierls instability coupled with an enharmonic phonon contribution [32]. The refractive index for $\mathrm{VO}_{2}$ at telecommunication and infrared regime is adapted from experimental results [33]. In telecommunication regime in the insulator phase, the refractive index is approximately $3+0.4 \mathrm{i}$, and it becomes $2+3 \mathrm{i}$ in the metallic phase [33]. Qualitatively, the effective dielectric function of $\mathrm{VO}_{2}$ at intermediate temperature is introduced by using Bruggeman effective medium theories [34]. Recently, several thermally controlled plasmonic structures based on $\mathrm{VO}_{2}$ have been proposed and experimentally demonstrated [31].

Passive plasmonic filters have been widely demonstrated theoretically and experimentally to achieve stop or pass wavelength selection [35, 36-37]. Active filters are mandatory to seamlessly integrate plasmonic components into a compact chip. Considerable efforts are dedicated in achieving tunable plasmonic filters [19, 38-41]. Recently, a tunable and highly efficient but slightly compact graphene-based band-stop filter composed of a periodic structure based on bilayer graphene nanoribbons in the mid-infrared region is demonstrated [38]. The filter is tuned over a wide range under applied voltage varying from $1 \mathrm{~V}$ to $5 \mathrm{~V}$.

In this paper, first, we studied a $\mathrm{VO}_{2}$-based waveguide (VIV) features under applied heat to achieve off and on states. The transmission is measured by a numerical approach based on the finite element method (FEM). Then, we added a FP resonator along the MIM waveguide to design a wide stopband filter and determined on/off transmission features. Finally, the structure is modified to operate as active passband in on/off states. Given the simple configuration and compact size, a wave guiding and filtering structure can be easily fabricated and highly integrated with other plasmonic devices on a chip.

\section{Structure description and theoretical model}

As shown in Figure 1, our proposed plasmonic waveguide is composed of two successive VIV and MIM waveguides, and p-polarized light is concentrated into the slit from Si-wire taper waveguide. The widths of VIV and MIM waveguides are equal and represented by W, and the length of VIV and MIM waveguides is represented by $\mathrm{h}$ and $\mathrm{L}$, respectively. The refractive index data of $\mathrm{VO}_{2}$ is adapted from the experimental work reported by Joushaghani et al. [33]. For telecommunication regime, the refractive index of insulator $\mathrm{VO}_{2}$ is approximately $3+0.4 \mathrm{i}$, whereas it is $\mathrm{n}=2+3 \mathrm{i}$ for the metallic $\mathrm{VO}_{2}$ [33]. The permittivity of silver is characterized by the Drude model:

$\varepsilon=\varepsilon_{\infty}-\frac{\omega_{p}^{2}}{\omega^{2}+i \omega \Gamma}$

where $\varepsilon_{\infty}, \omega_{\mathrm{p}}$, and $\Gamma$ stand for high-frequency bulk permittivity, bulk plasmon frequency, and electron collision frequency, respectively, which are set as $4.2,1.346 \times 10^{16} \mathrm{rad} / \mathrm{s}$, and $9.617 \times 10^{13}$ $\mathrm{rad} / \mathrm{s}$ [42]. The insulator layer operates as a core; thus, narrower cores have stronger confinement. When $\mathrm{VO}_{2}$ is in the metallic phase, for cores smaller than the incident wavelength, only one propagation mode is identified [43], where its propagation constant $(\beta)$ can be determined by solving the dispersion relation [43, 44]:

$$
k_{V \cdot m}+\varepsilon_{V \cdot m} k_{d} \tanh \left(\frac{k_{d} w}{2}\right)=0
$$


where $\mathrm{k}_{\mathrm{m}}$ and $\mathrm{k}_{\mathrm{d}}$ are defined as $k_{d}=\left(\beta^{2}-k_{0}^{2}\right)^{1 / 2}$ and $k_{V \cdot m}=\left(\beta^{2}-\varepsilon_{V . m} k_{0}^{2}\right)^{1 / 2}$, and $\mathrm{k}_{0}=2 \pi / \lambda$ is the free space wave vector, and $\varepsilon_{V}$ is the permittivity of $\mathrm{VO}_{2}$ in the metallic phase. Light experiences larger refractive index inside the waveguide because of light squeezing and other observed phenomena, which is known as the effective refractive index. The fabrication process is straightforward; first, the silver layer is deposited on the substrate by sputtering technique, and then the $\mathrm{VO}_{2}$ film is attached by using lithography or deposition techniques. Finally, the slit is perforated by using ion beam milling.

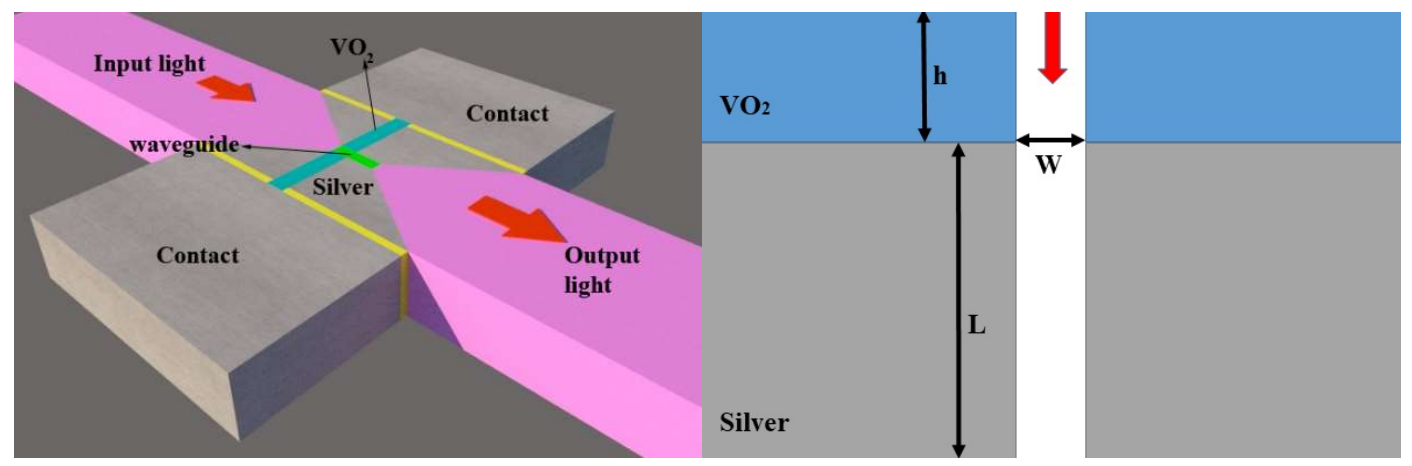

Fig.1 Schematic of the proposed structure. W,h, L stands for waveguide width, VIV waveguide length and MIM waveguide length, respectively. The P-polarized incident light is coupled to the waveguide from Si nanowire taper.

\section{Simulation results and analysis}

In the simulation, the fundamental TM mode of the waveguide is excited by a pulse dipole source from the taper on the left and coupled to the waveguide in the $\mathrm{VO}_{2}$ medium. $P$ and $Q$ are set as the entrance and exit ports of waveguide to detect the transmitted powers of $P_{\text {in }}$ and $P_{t r}$ and calculate the transmission of two power monitors. Transmission is defined as $T=P_{t r} / P_{i n}$. The absorption parameter, which is a measurement of dissipation of the power in the device, can be simply deduced by $A=1-R-T$, where $\mathrm{R}$ is the reflection. The geometrical parameters of the structure are set to be $\mathrm{W}=150 \mathrm{~nm}, \mathrm{~h}=600 \mathrm{~nm}$, and $\mathrm{L}=1100 \mathrm{~nm}$. When the structure is in off state (no heating is applied), light transmission through the waveguide is negligible. For insulator $\mathrm{VO}_{2}$, the VIV waveguide becomes a dielectric waveguide because near refractive indexes of the media light cannot confine and propagate through it. When heat is applied to the structure, $\mathrm{VO}_{2}$ undergoes a transition and becomes metal, and light properly propagates through it. Figure 2(a) depicts the transmission in the insulator and metal phases. It is seen that for wide wavelength range, the transmission in the metal state is considerably high, whereas in the insulator state, the transmission dropped notably. Fig. 2 (b, c) represents the magnetic field distribution in both metallic (ON) and insulator (OFF) states. In the metallic state, light propagates normally, whereas in the insulator state, light is not confined inside the waveguide, and it propagates in the entire $\mathrm{VO}_{2}$ medium. 

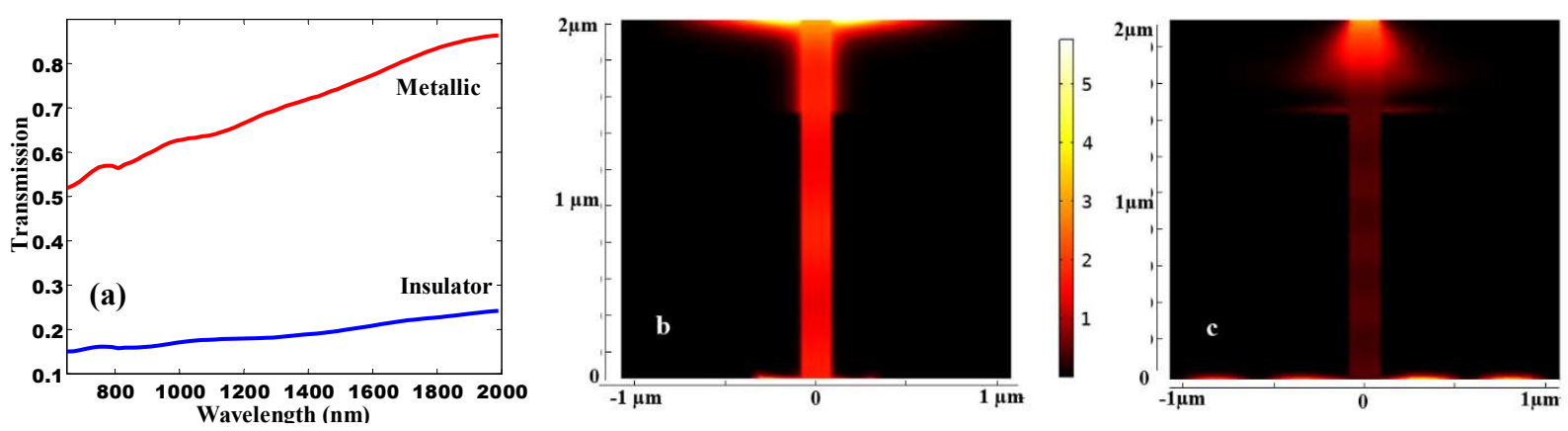

Fig 2. (a) Transmission profile when $\mathrm{VO}_{2}$ is in metallic $(\mathrm{ON})$ and insulator $(\mathrm{OFF})$ phase. (b) Magnetic field distribution when $\mathrm{VO}_{2}$ is in metallic phase. (c) Magnetic field distribution when $\mathrm{VO}_{2}$ is in insulator phase.

The length of the VIV and MIM layers can affect the transmission features. The longer the VIV, the more dissipation occurs and the lower the transmission. Figs. 3(a,b) show the transmission profile versus different VIV and MIM lengths. Longer VIV decreases the transmission in the metallic and insulator phases. Based on our requirement, suitable compromising should be conducted. By contrast, the transmission is not affected notably by the MIM length. However, MIM waveguides larger than the SPP propagation length because of inherent loss of metal will decrease the transmission. Fig. 3(c) shows the transmission profile versus the waveguide width. The narrower the waveguides, the less power is transmitted; hence, less transmission occurs.
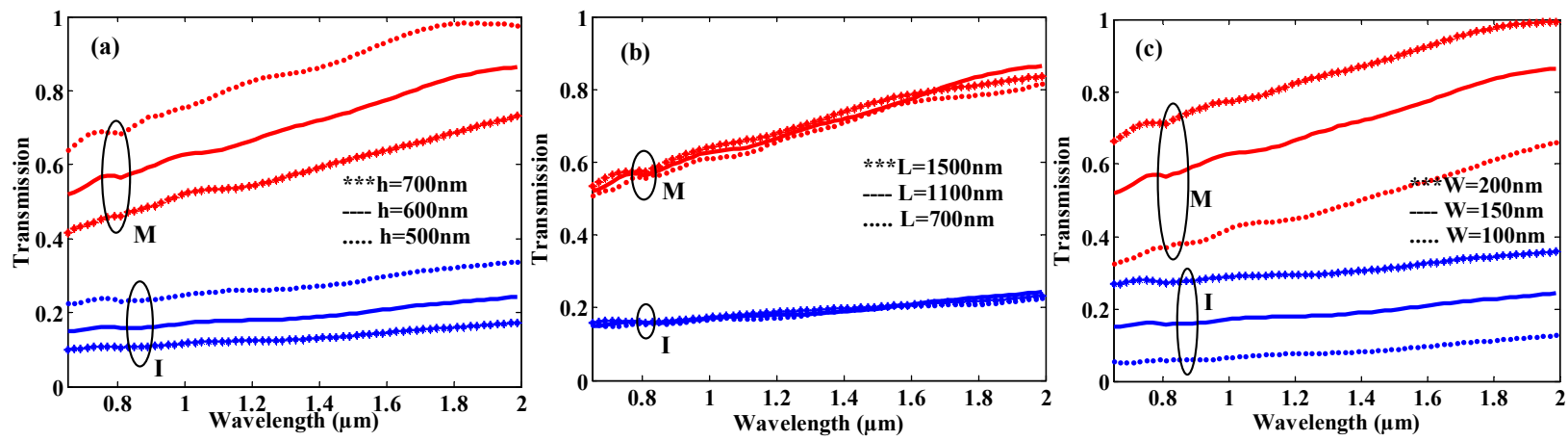

Fig 3. Spectral dependence of transmission for various (a) $\mathrm{VO}_{2}$ waveguide length, (b) Metallic waveguide Length, and (c) waveguide width. It can be seen that MIM length doesn't affect the transmission.

In optical chips light transmission at a specific wavelength be blocked. Different configuration has been proposed as passive stopband structures. Most popular structures are designed by employing FP cavities. If the FP cavity locates in the vicinity of a waveguide, then it can confine portion of the propagating light within the waveguide. This light confinement merely occurs at some specific wavelengths corresponding to resonance wavelength of the cavity. The resonant condition that can determine the resonant wavelength can be determined through the following equation [45]:

$2 \frac{2 \pi}{\lambda_{m}} \operatorname{Re}\left(N_{\text {eff }}\right) d+\Delta \phi=2 m \pi$

where $\mathrm{d}$ is the FP cavity length; $\mathrm{N}_{\text {eff }}$ is the effective refractive index of the cavity, and $\Delta \Phi$ is the phase shift of the beam, which experiences reflection on the two end facets of the cavity. The positive integer $m$ represents the number of antinodes of the standing wave. $\lambda_{m}$ straightforwardly can be calculated using equation 3. We embedded the FP cavity (Fig. 4[a]) in our waveguide structure to obtain an active stop band filter. $d$ and $t$ stand for cavity length and width, respectively, 
which are set as 580 and $150 \mathrm{~nm}$, respectively, whereas the gap is fixed at $10 \mathrm{~nm}$. Fig. 4 (b) depicts the transmission profile; when heat is applied, the $\mathrm{VO}_{2}$ becomes metal. Two resonances corresponding to the resonance wavelength of the cavity are distinct on the plot. For these resonances, the transmission drops sharply. The blue line shows the transmission when heating is $\mathrm{OFF}$ and $\mathrm{VO}_{2}$ is in the insulator phase. Magnetic field distributions for resonance wavelength in the metallic and insulator phases of $\mathrm{VO}_{2}$ are presented in Figs. 4(c, d). Large portion of light trapped within the cavity and standing waves is created.
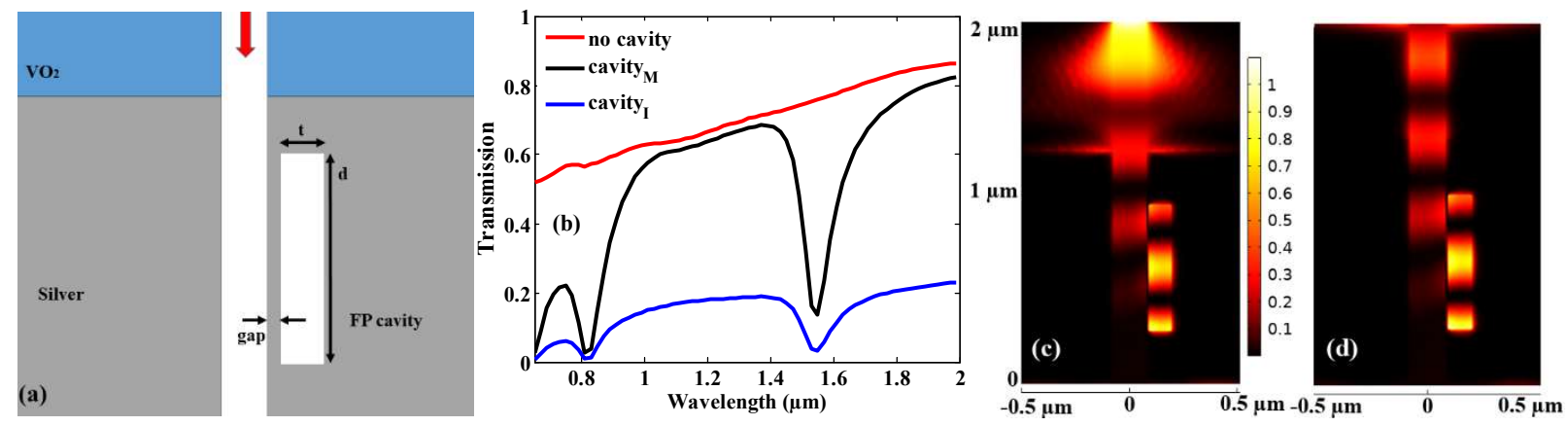

Fig 4. (a) 2D schematic of the proposed stop band filter. $t$ and $d$ stands for cavity length and width. The gap is considered to be $10 \mathrm{~nm}$ to ensure noticeable coupling. (b) transmission profile when $\mathrm{VO}_{2}$ is in metallic and insulator phase. (c) Magnetic field distribution when $\mathrm{VO}_{2}$ is in insulator mode (d) Magnetic field distribution when $\mathrm{VO}_{2}$ is in metallic mode.

It is worthy to note that the resonance wavelengths directly depend on the cavity length (d), and the effective refractive index indirectly depends on the cavity width $(\mathrm{t})$. Therefore, wavelength tuning can be performed through tailoring geometrical parameters of the cavity. The gap between the cavity and the waveguide is a critical parameter that can affect transmission intensity. Fig. 5 (a) shows the transmission profile versus the cavity length, whereas Fig. 5(b) shows the transmission versus the cavity width. It can clearly be seen that there is a red-shift of the transmission spectrum as the length and width of cavity increases. Further, by the increasing the length and width of cavity, the transmission intensity increases. For any desired resonance wavelength, the appropriate geometrical parameters can be adjusted. The penetration depth of SPP into silver is estimated to be less than $30 \mathrm{~nm}$; therefore, considerable coupling the gap should be less than the penetration depth. Fig. 5 (c) shows the gap thickness dependence of transmission. For narrower gaps, the cavity can trap the light considerably. The wider the gap, the less the light confinement.
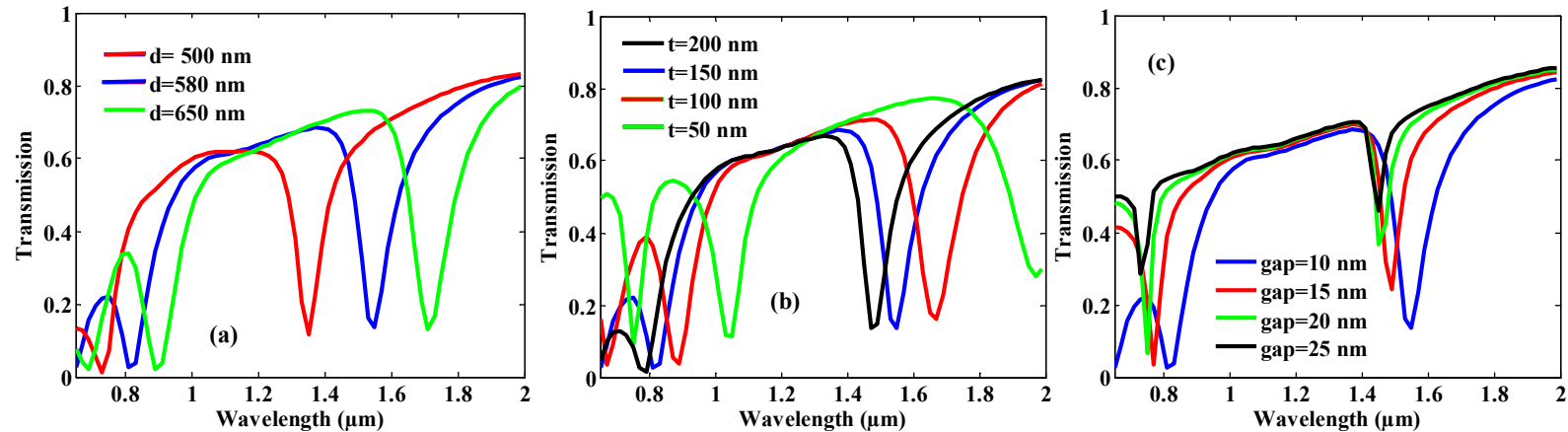

Fig 5. (a) Spectral dependence of transmission for various (a) FP cavity length, (b) FP cavity width, and (c) the gap between cavity and waveguide.

In the next step, we modified our structure to design a passband filter. We utilized the FP cavity in the waveguide to permit light propagation in specific wavelengths. The structure is shown in 
Fig. 6 (a). The FP cavity is embedded inside the waveguide with two narrow separating gaps. The resonance condition and propagation mechanism are similar to the stopband filter, which can be estimated using equation 3. In the transmission profile depicted in Fig. 6(b), two peaks correspond to the resonance wavelength of the cavity can be seen. The black line shows the transmission when $\mathrm{VO}_{2}$ is in the metallic phase, whereas the blue line indicates transmission in the insulator phase. The red line shows the transmission when no cavity is presented. In general, the presence of cavities leads to light propagation and decreases the transmission. Fig. 6(c) shows the magnetic field distribution in resonance wavelength and the standing waves inside the cavity.
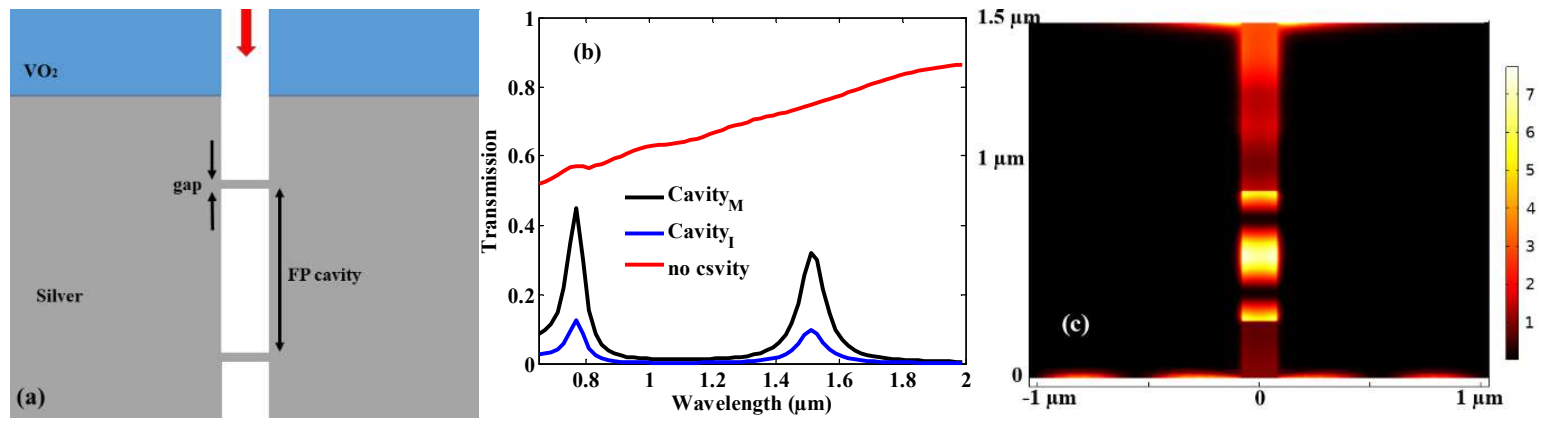

Fig 6. (a) 2D schematic of the proposed passband filter. The gap is considered to be $10 \mathrm{~nm}$ to ensure noticeable coupling. (b) transmission profile when $\mathrm{VO}_{2}$ is in metallic and insulator phase. (c) Magnetic field distribution when $\mathrm{VO}_{2}$ is in metallic mode.

The above mentioned structure based on geometrical parameters has various resonances. Another configuration also shows stopband behavior (Fig. 7[a]). This structure has features that depend on input-output location, which can permit only one resonance coupling to the output port (Fig. 7[b]) and field distribution (Fig. 7[c]). Fei investigated the transmission behavior by the arbitrary input position and superposition of magnetic fields inside the cavity and determined in what input positions single resonance can be seen $[45,46]$. When input is at the center of the cavity, only first resonance will be presented (B point). For locations, where the input port is on both end sides of the cavity, both resonances will occur (A and $\mathrm{C}$ ). However, if the input moves from the center about $\mathrm{d} / 4$, then the second resonance will be propagated. Moreover, by placing two outputs in different locations, the propagation at specific wavelength through the particular output can occur.
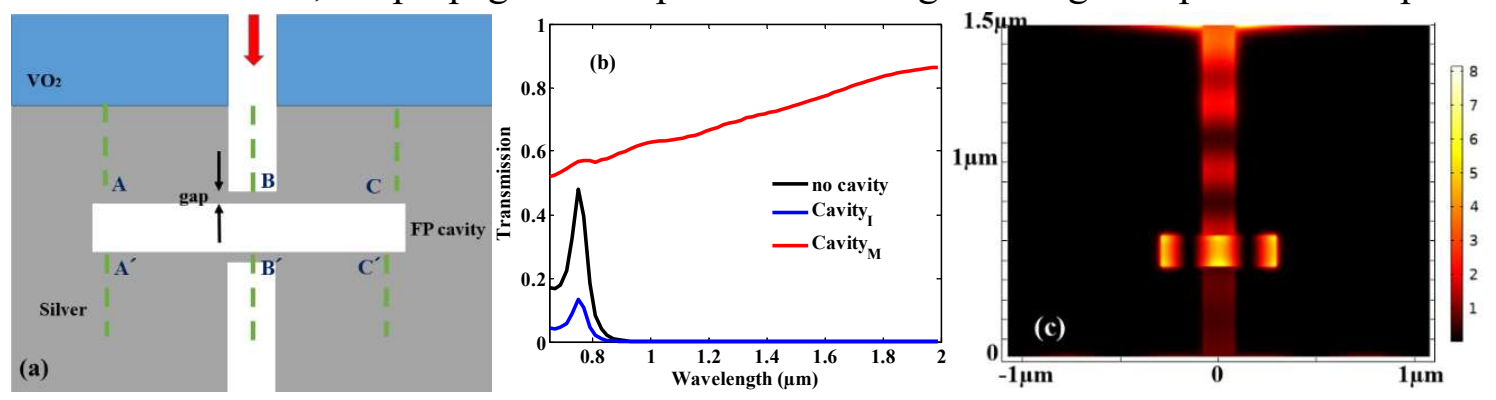

Fig 7. (a) 2D schematic of the proposed passband filter. The gap is considered to be $10 \mathrm{~nm}$ to ensure noticeable coupling. (b) transmission profile when $\mathrm{VO}_{2}$ is in metallic and insulator phase. (c) Magnetic field distribution when $\mathrm{VO}_{2}$ is in metallic mode.

The two above mentioned configurations despite their lower transmission present a narrow transmission profile. The output port is modified to increase the transmission to two locations of the cavity (Fig. 8[a]). Ports and cavity are in parallel and located in a way that the bottom of the input port axes is under the cavity's center. In addition, top of output port is above the center of the cavity. This difference in location is represented by $\Delta \mathrm{L}$ in the picture and set as $250 \mathrm{~nm}$. The width of the input, cavity, and output ports are represented by $\mathrm{W}_{1}, \mathrm{~W}_{2}$, and $\mathrm{W}_{3}$, respectively. In 
general, $\mathrm{W}_{1}$ and $\mathrm{W}_{3}$ are equal to the modified width of the cavity. The transmission profile is depicted in Fig. 8(b). The transmission (black line) output widths, when $\mathrm{W}_{2}$ is equal to the input, are increased slightly; however, the profile is broadened and red-shifted. Green and $\mathrm{C}$ lines show the transmission when $\mathrm{W}_{2}$ is set as 100 and $50 \mathrm{~nm}$. According to the resonance condition of the cavity, any changes in the cavity width will result in different effective refractive indices and correspondingly different resonance wavelengths. Furthermore, the narrow cavities have less coupling. Fig. 8(c) shows the magnetic field distribution at resonance wavelength. Standing waves inside the cavity can be seen.

According to our experimental obstacles, proper arrangement can be considered, and desired resonance wavelength can be tuned.
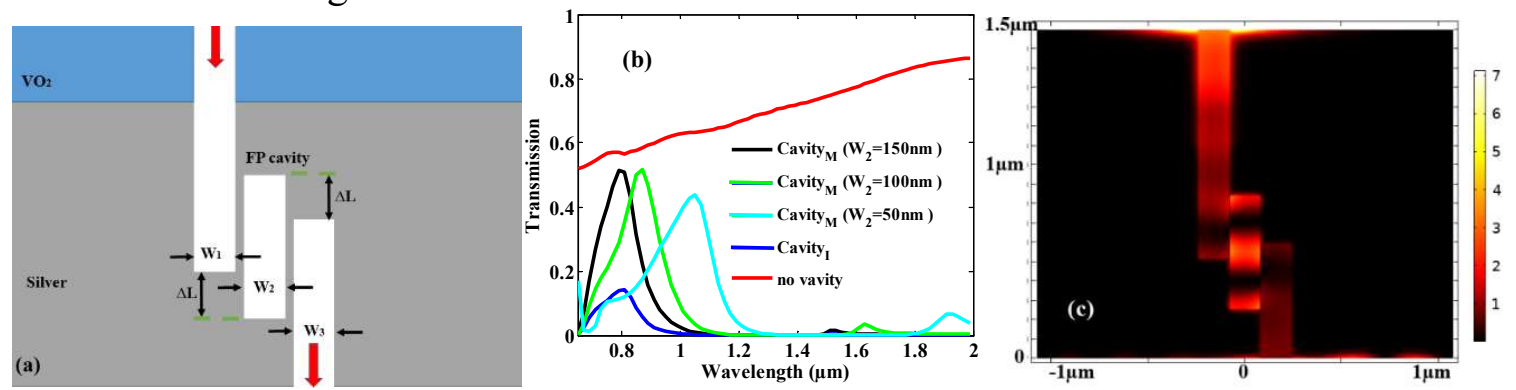

Fig 8. (a) 2D schematic of the proposed passband filter. The gap is considered to be $10 \mathrm{~nm}$ to ensure noticeable coupling. (b) transmission profile when $\mathrm{VO}_{2}$ is in metallic and insulator phase. (c) Magnetic field distribution when $\mathrm{VO} 2$ is in metallic mode.

\section{Conclusion}

In conclusion, an active subwavelength plasmonic waveguide, stopband, and passband filter are proposed by using PCM and Fabry Perot cavity and theoretically and numerically investigated. $\mathrm{ON}$ and OFF states that are created by introducing heat to the system showed two distinct features when $\mathrm{VO}_{2}$ was in the metallic or insulator state. The role of the geometrical parameters is determined in wave guiding features. Furthermore, the filtering characteristics of both filters are studied, and different arrangements based on the location of input, output, and cavity are also presented. Given this simple configuration, the structure has high potential and feasibility to be integrated in nanoscale plasmonic chips and circuits.

\section{Declarations}

\section{Funding}

The authors did not receive support from any organization for the submitted work.

\section{Conflicts of interest}

The authors have no conflicts of interest to declare that are relevant to the content of this article.

\section{Availability of data and material}

The data presented in this study are available on request from the corresponding author.

\section{Code availability}

The code used in this study are available on request from the corresponding author. 


\section{Authors' contributions}

The corresponding author is responsible for ensuring that the descriptions are accurate and agreed by all authors.

\section{Ethics approval}

Not applicable.

\section{Consent to participate}

Not applicable.

\section{Consent for publication}

Not applicable.

\section{References}

[1] S. A. Maier, Plasmonics: fundamentals and applications. Springer Science \& Business Media, 2007.

[2] Anatoly V. Zayats Stefan A. Maier, Active Plasmonics and Tuneable Plasmonic. John Wiley \& Sons, Inc, 2013.

[3] M. Afshari-Bavil, M. Dong, C. Li, S. Feng, and L. Zhu, "Thermally Controllable HighEfficiency Unidirectional Coupling in a Double-Slit Structure Filled with Phase Change Material," IEEE Photonics J.,11,no. 2, pp. 1-8, 2019.

[4] M. A. Bavil, Q. Deng, and Z. Zhou, "Extraordinary transmission through gain-assisted silicon-based nanohole arrays in telecommunication regimes.," Opt. Lett., vol. 39, no. 15, pp. 4506-9, 2014.

[5] X. Wang, Z. Cheng, K. Xu, H. K. Tsang, and J.-B. Xu, "High-responsivity graphene/siliconheterostructure waveguide photodetectors," Nat. Photonics, vol. 7, no. 11, pp. 888-891, 2013.

[6] E. Bermúdez-Ureña et al., "Plasmonic Waveguide-Integrated Nanowire Laser," Nano Lett., vol. 17, no. 2, pp. 747-754, 2017.

[7] A. Joushaghani, J. Jeong, S. Paradis, D. Alain, J. Stewart Aitchison, and J. K. S. Poon, "Wavelength-size hybrid Si-VO_2 waveguide electroabsorption optical switches and photodetectors," Opt. Express, vol. 23, no. 3, p. 3657, 2015.

[8] K. J. Miller, K. A. Hallman, R. F. Haglund, and S. M. Weiss, "Silicon waveguide optical switch with embedded phase change material," Opt. Express, vol. 25, no. 22, p. 26527, 2017.

[9] H. A. L. Iang, R. I. S. Oref, J. M. U. Ianwei, and X. L. I. Un, "Using Three- and FourWaveguide Directional Couplers," Appl. Opt., vol. 54, no. 19, 2015.

[10] W. Cai, W. Shin, S. Fan, and M. L. Brongersma, "Elements for plasmonic nanocircuits with three-dimensional slot waveguides," Adv. Mater., vol. 22, no. 45, pp. 5120-5124, 2010.

[11] M. Ono, H. Taniyama, and E. Kuramochi, "Toward Application of Plasmonic Waveguides to Optical Devices," pp. 14-19.

[12] M. Mirahmadi, T. Mahinroosta, and S. M. Hamidi, "Manipulating plasmon-exciton interactions in the plasmonic waveguide structure based on the dispersion relations concept," Opt. Quantum Electron., vol. 52, no. 6, pp. 1-9, 2020. 
[13] F. Yang et al., "Ultrathin Broadband Germanium-Graphene Hybrid Photodetector with High Performance," ACS Appl. Mater. Interfaces, vol. 9, no. 15, pp. 13422-13429, 2017.

[14] E. Fondamentale, U. M. R. Cnrs, S. Xi, and O. Cedex, Compound Semiconductor Nanowire Photodetectors, 1st ed. Elsevier Inc., 2015.

[15] M. Sun, W. Shieh, and R. R. Unnithan, "Design of plasmonic modulators with vanadium dioxide on silicon-on-insulator," IEEE Photonics J., vol. 2017-Janua, no. 3, pp. 77-85, 2017.

[16] G. Zhou et al., "Broadband and high modulation-depth THz modulator using low bias controlled VO_2-integrated metasurface," Opt. Express, vol. 25, no. 15, p. 17322, 2017.

[17] R. Thomas, Z. Ikonic, and R. W. Kelsall, "Electro-optic metal-insulator-semiconductorinsulator-metal Mach-Zehnder plasmonic modulator," Photonics Nanostructures - Fundam. Appl., vol. 10, no. 1, pp. 183-189, 2012.

[18] J. K. Clark, Y. L. Ho, H. Matsui, and J. J. Delaunay, "Optically Pumped Hybrid PlasmonicPhotonic Waveguide Modulator Using the VO2Metal-Insulator Phase Transition," IEEE Photonics J., vol. 10, no. 1, 2018.

[19] L. Trimby, A. Baldycheva, and C. D. Wright, "Phase-change band-pass filters for multispectral imaging," SPIE Proc., vol. 10541, pp. 105412B-10541-8, 2018.

[20] M. A. Bavil, X. Sun, and F. Huang, "Classifying the transmission resonances of a subwavelength aperture within a thin metallic film by breaking the symmetry," Phys. B Condens. Matter, vol. 407, no. 14, 2012.

[21] A. Foroughifar, H. Saghaei, and E. Veisi, "Design and analysis of a novel four-channel optical filter using ring resonators and line defects in photonic crystal microstructure," Opt. Quantum Electron., vol. 53, no. 2, pp. 1-12, 2021.

[22] A. B. Asl, A. Rostami, and I. S. Amiri, "Terahertz band pass filter design using multilayer metamaterials," Opt. Quantum Electron., vol. 52, no. 3, pp. 1-13, 2020.

[23] M. Afshari Bavil, C. Li, S. Feng, M. Dong, and L. Zhu, "Compact plasmonic lens based on gradually decreasing size and separation nanohole cluster," Journal of Optics 21, no. 8 pp. 085001.2019.

[24] G. H. Yuan, E. T. Rogers, and N. I. Zheludev, "Achromatic super-oscillatory lenses with sub-wavelength focusing," Light Sci. Appl., vol. 6, no. 9, pp. e17036-8, 2017.

[25] C. Lu, X. Hu, H. Yang, and Q. Gong, "Chip-integrated ultrawide-band all-optical logic comparator in plasmonic circuits," pp. 1-8, 2014.

[26] A. Emboras et al., "Electrically Controlled Plasmonic Switches and Modulators," IEEE Journal of Selected Topics in Quantum Electronics 21, no. 4, pp. 276-283, 2014.

[27] Ding, Y., Guan, X., Zhu, X., Hu, H., Bozhevolnyi, S.I., Oxenløwe, L.K., Jin, K.J., Mortensen, N.A. and Xiao, S., "Effective electro-optic modulation in low-loss grapheneplasmonic slot waveguides," Nanoscale, 9, no. 40, pp.15576-15581, 2017.

[28] K. S. Karimov, M. Saleem, N. Ahmad, and A. Rashid, "Optical transmission in thin films of vanadium compounds," vol. 35, no. 7, pp. 3-7, 2014.

[29] K. S. Karimov, M. Saleem, M. T. S. Chani, and A. K. Niaz, "Temperature sensor based on composite film of vanadium complex ( VO 2 ( 3-fl )) and CNT," vol. 36, no. 7, pp. 1-5, 2015.

[30] L. Juntao, L. Bo, S. Zhitang, Y. Dongning, and F. Songlin, "Reactive ion etching of Si 2 Sb 2 Te 5 in CF 4 / Ar plasma for a nonvolatile phase-change memory device," vol. 34, no. 5, pp. 4-8, 2013.

[31] H. Kocer et al., "Thermal tuning of infrared resonant absorbers based on hybrid goldVO2nanostructures," Appl. Phys. Lett., vol. 106, no. 16, 2015. 
[32] J. D. Budai et al., "Metallization of vanadium dioxide driven by large phonon entropy," Nature, vol. 515, no. 7528, p. 535, 2014.

[33] A. Joushaghani, "Arash Joushaghani A thesis submitted in conformity with the requirements for the degree of Doctorate of Philosophy Graduate Department of Electrical and Computer Engineering c Copyright 2014 by Arash Joushaghani Abstract," Thesis, 2014.

[34] P. U. Jepsen et al., "Metal-insulator phase transition in a V O2 thin film observed with terahertz spectroscopy," Phys. Rev. B - Condens. Matter Mater. Phys., vol. 74, no. 20, 2006.

[35] M. A. A. Bavil, L. Gao, and X. Sun, "A Compact Nanoplasmonics Filter and Intersection Structure Based on Utilizing a Slot Cavity and a Fabry-Perot Resonator," Plasmonics, vol. 8, no. 2, pp. 631-636, 2013.

[36] B. Yun, G. Hu, and Y. Cui, "A nanometric plasmonic waveguide fi lter based on Fabry Perot resonator," OPTICS, vol. 284, no. 1, pp. 485-489, 2011.

[37] P. F. Mcmanamon et al., "Optical phased array technology," Proc. IEEE, vol. 84, no. 2, pp. 268-298.

[38] X. Wang et al., "Tunable graphene-based mid-infrared plasmonic multispectral and narrow band-stop filter" Label Free urea Biosens. based Org. Electrochem. transistors, pp. 0-15, 2018.

[39] W. Li, S. jiang Chang, X. hui Wang, L. Lin, and J. jun Bai, "A thermally tunable terahertz bandpass filter with insulator-metal phase transition of VO2thin film," Optoelectron. Lett., vol. 10, no. 3, pp. 180-183, 2014.

[40] Y. Zhu et al., "Tunable dual-band terahertz metamaterial bandpass filters.," Opt. Lett., vol. 38, no. 14, pp. 2382-4, 2013.

[41] K. J. A. Ooi, P. Bai, H. S. Chu, and L. K. Ang, "Regular article Ultracompact vanadium dioxide dual-mode plasmonic waveguide electroabsorption modulator," vol. 2, no. 1, pp. 13-19, 2013.

[42] K. L. Shuford, M. A. Ratner, S. K. Gray, and G. C. Schatz, "Finite-difference time-domain studies of light transmission through nanohole structures," Appl. Phys. B, vol. 84, no. 1-2, pp. 11-18, 2006.

[43] J. A. Dionne, L. A. Sweatlock, H. A. Atwater, and A. Polman, "Plasmon slot waveguides: Towards chip-scale propagation with subwavelength-scale localization," Phys. Rev. B, vol. 73, no. 3, p. 35407, 2006.

[44] B. Wang and G. P. Wang, "Plasmon Bragg reflectors and nanocavities on flat metallic surfaces," Appl. Phys. Lett., vol. 87, no. 1, p. 13107, 2005.

[45] F. Hu, H. Yi, and Z. Zhou, "Band-pass plasmonic slot filter with band selection and spectrally splitting capabilities," Opt. Express, vol. 19, no. 6, pp. 24096-24101, 2011.

[46] S. A. Akhmanov and S. Y. Nikitin, Physical optics. Clarendon Press, 1997. 


\section{Figures}

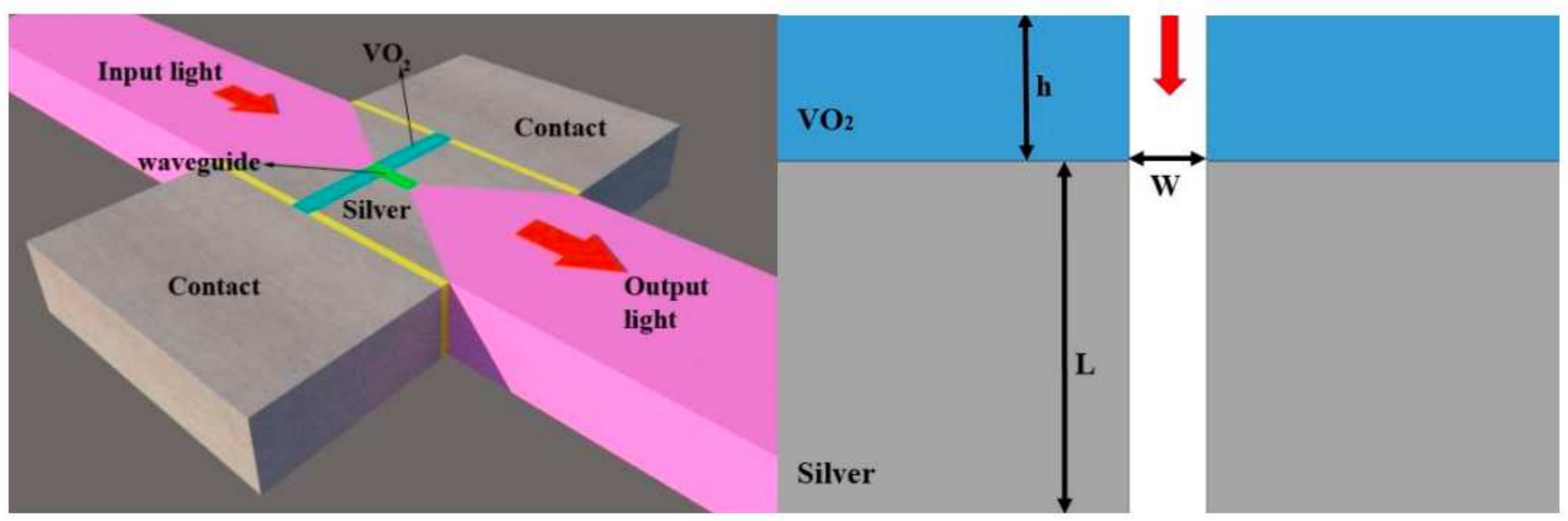

\section{Figure 1}

Schematic of the proposed structure. W, h, L stands for waveguide width, VIV waveguide length and MIM waveguide length, respectively. The P-polarized incident light is coupled to the waveguide from $\mathrm{Si}$ nanowire taper.
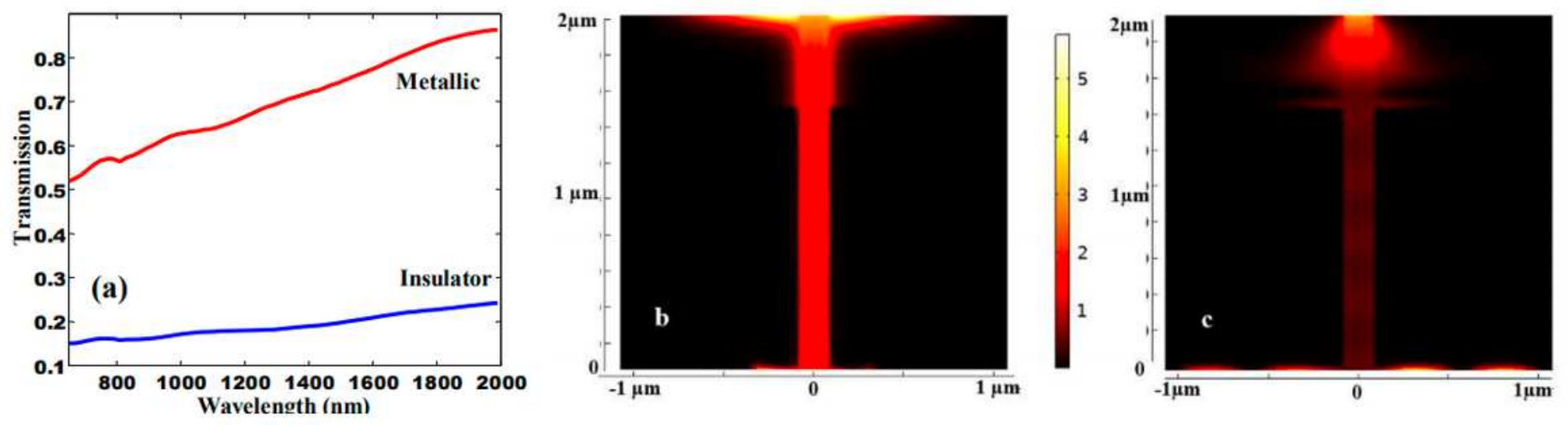

Figure 2

(a) Transmission profile when VO2 is in metallic (ON) and insulator (OFF) phase. (b) Magnetic field distribution when $\mathrm{VO} 2$ is in metallic phase. (c) Magnetic field distribution when VO2 is in insulator phase. 

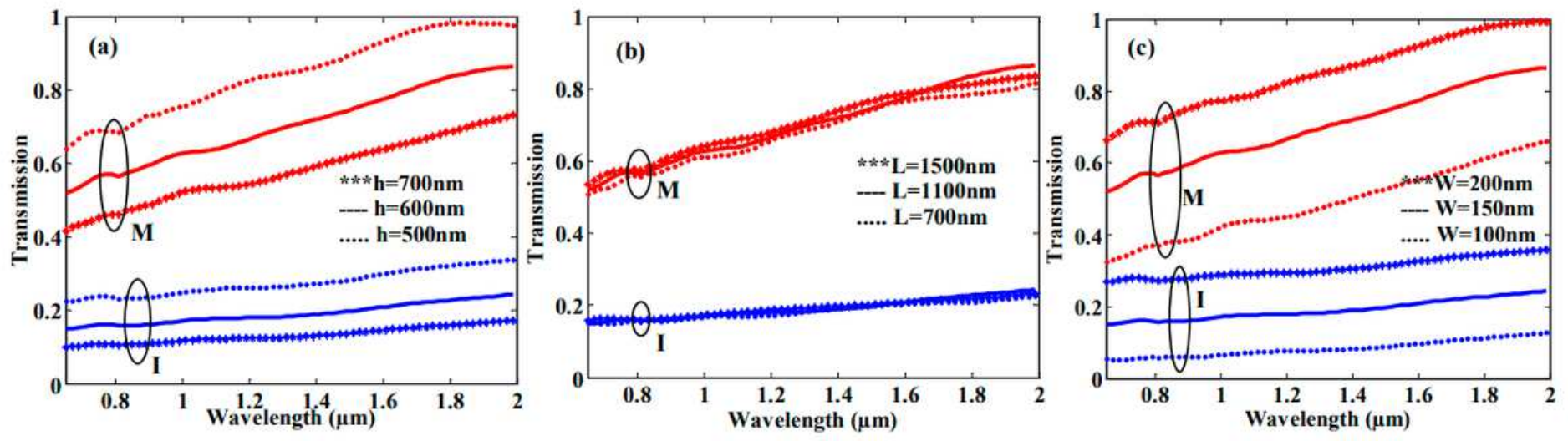

Figure 3

Spectral dependence of transmission for various (a) VO2 waveguide length, (b) Metallic waveguide Length, and (c) waveguide width. It can be seen that MIM length doesn't affect the transmission.
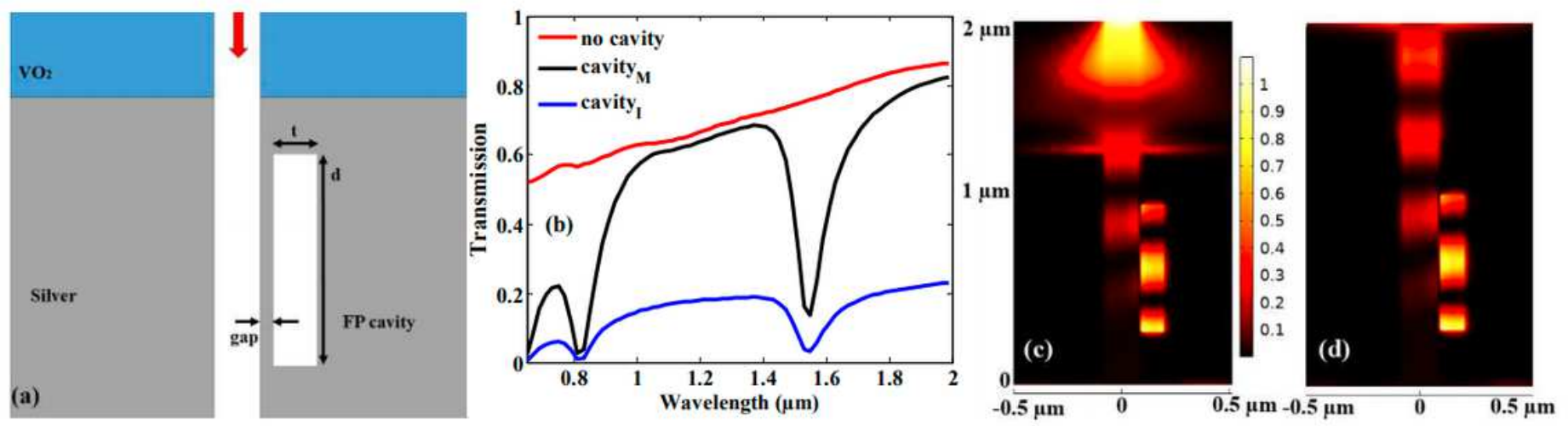

\section{Figure 4}

(a) 2D schematic of the proposed stop band filter. $t$ and $d$ stands for cavity length and width. The gap is considered to be $10 \mathrm{~nm}$ to ensure noticeable coupling. (b) transmission profile when VO2 is in metallic and insulator phase. (c) Magnetic field distribution when VO2 is in insulator mode (d) Magnetic field distribution when VO2 is in metallic mode.
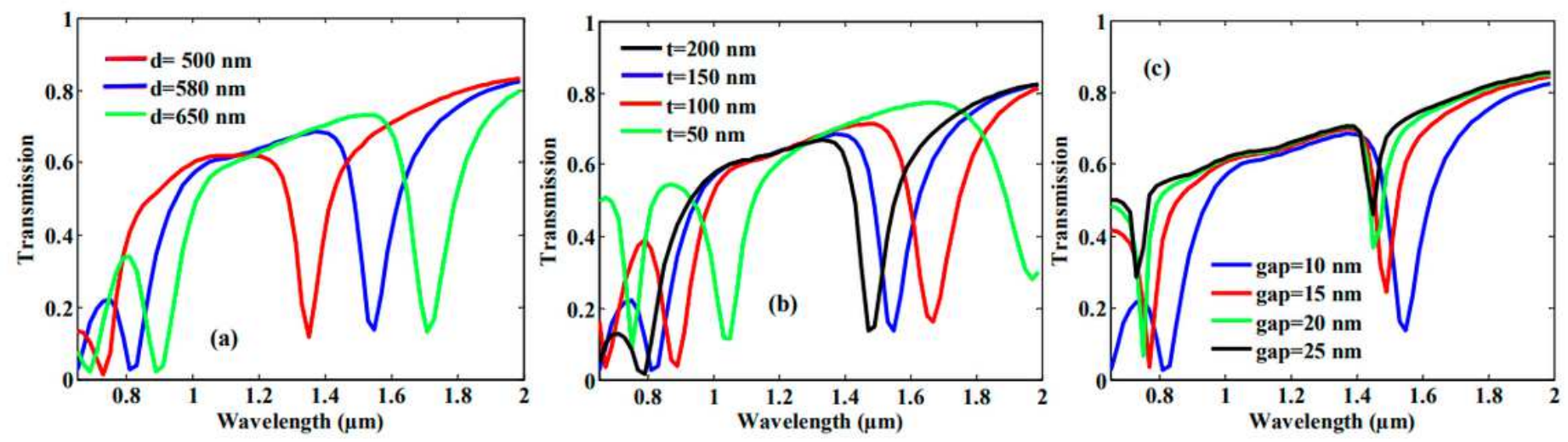

Figure 5 
(a) Spectral dependence of transmission for various (a) FP cavity length, (b) FP cavity width, and (c) the gap between cavity and waveguide.
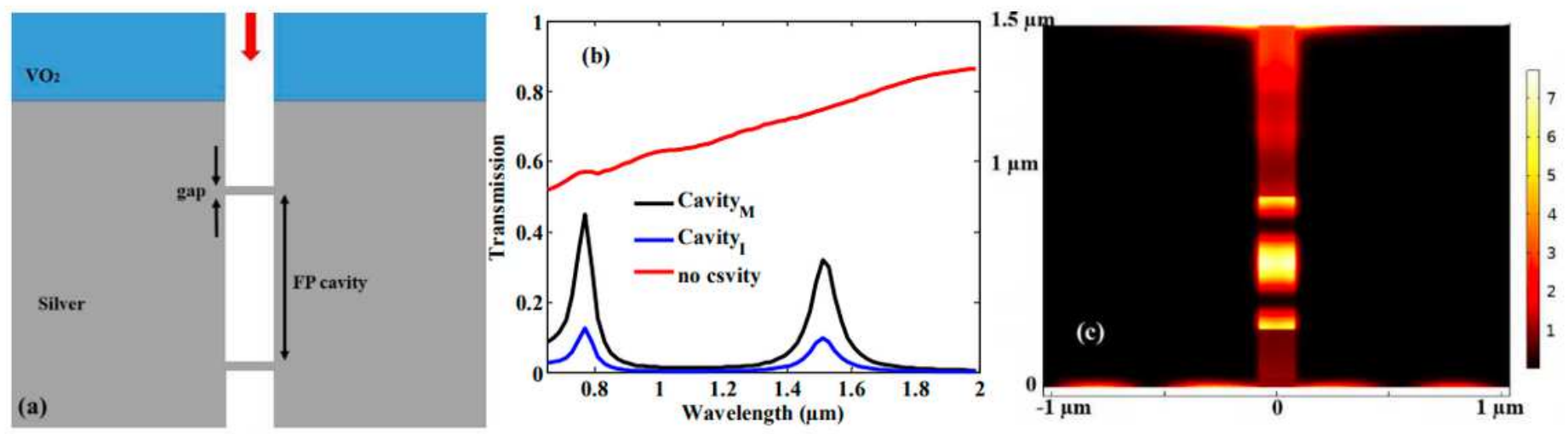

\section{Figure 6}

(a) 2D schematic of the proposed passband filter. The gap is considered to be $10 \mathrm{~nm}$ to ensure noticeable coupling. (b) transmission profile when VO2 is in metallic and insulator phase. (c) Magnetic field distribution when $\mathrm{VO} 2$ is in metallic mode.
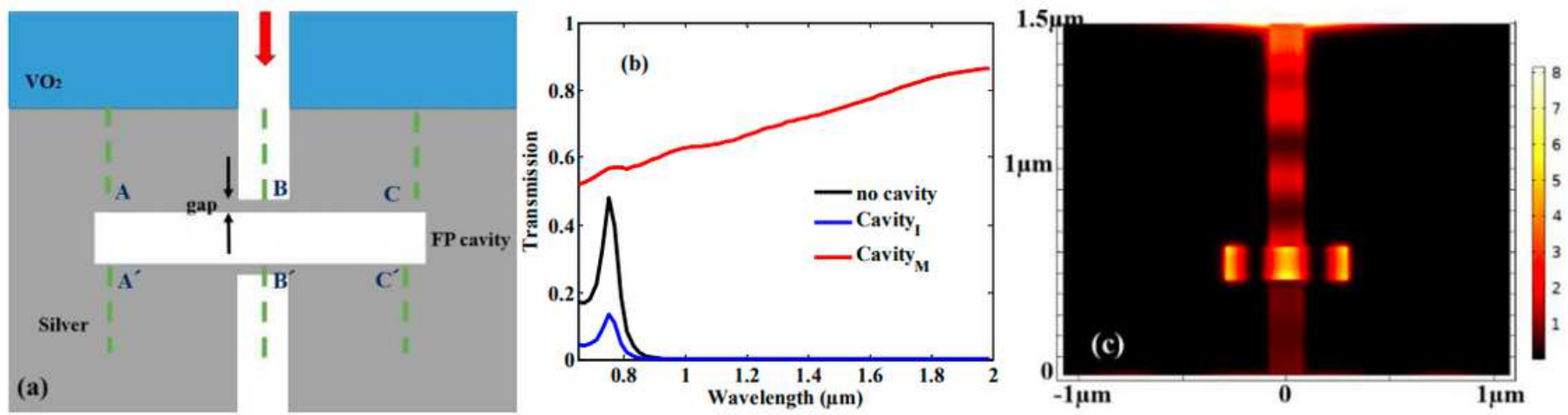

\section{Figure 7}

(a) 2D schematic of the proposed passband filter. The gap is considered to be $10 \mathrm{~nm}$ to ensure noticeable coupling. (b) transmission profile when VO2 is in metallic and insulator phase. (c) Magnetic field distribution when $\mathrm{VO} 2$ is in metallic mode.
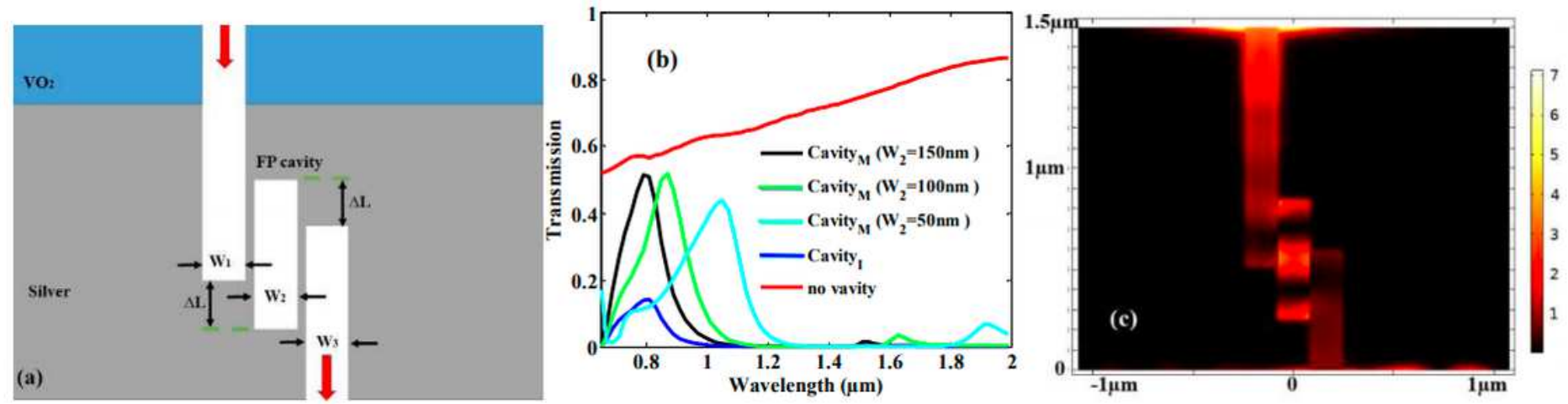
(a) 2D schematic of the proposed passband filter. The gap is considered to be $10 \mathrm{~nm}$ to ensure noticeable coupling. (b) transmission profile when VO2 is in metallic and insulator phase. (c) Magnetic field distribution when VO2 is in metallic mode. 